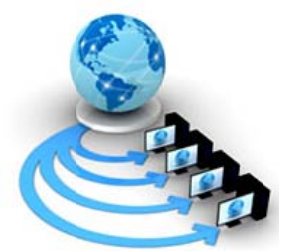

Volume 9, No. 1, January-February 2018

International Journal of Advanced Research in Computer Science

REVIEW PAPER

\title{
TOWARDS GREEN COMPUTING, IMPORTANCE, IMPACT AND POSSIBLE SOLUTIONS-A REVIEW
}

\author{
Ambooj Yadav \\ Department of Computer Science and Engineering, \\ JamiaHamdard, New Delhi, India-110062
}

\author{
Bhavya Alankar ${ }^{2}$ \\ Department of Computer Science and Engineering, \\ JamiaHamdard, New Delhi, India-110062
}

\begin{abstract}
Fresh environment is the key to human life, according to Business today report 25 lakh people died only in India till 2015 due to the polluted atmosphere. Major causes of air pollution are burning of fossil fuels, agricultural activities, exhaust from factories and industries, mining operations, and indoor air pollution. Unfortunately, our beloved computer is one of the among. Datacenters discharge massive amount of $\mathrm{CO}_{2}$ in the air as indatacenters thousands of servers, and other computer components are continuously working $24 \times 7$. It occurs to researchers that they must search some new ways to control this problem. That's why green computing is a trending topic today. In this paper, we have discussed the importance of green computing or green cloud computing, the impact of greenhouse gases on nature, also we will suggest some ideas that could be implemented in datacenters.
\end{abstract}

Keyword:-Cloud Computing, Green Cloud, Recycling, Performance Matrix, Green House Gases.

\section{INTRODUCTION}

From the past a decade or two cloud computing is the backbone for all the institutions, organizations and government departments, whether big or small. The cloud provides tremendous features and facilities like easy to access, pay and use model, security, scalability, anywhere any time availability and much more. To see the growing demand of cloud computing it was necessary that we make such places where we install high tech computers and unimaginable amount of storage to provide cloud services in much cheaper rates than owning computers and high expenditure, these places known as the Datacenters, with time thousands of datacentres came into existence. When the number of datacentre increased, naturally electrical consumption came into limelight, so heating issue was obvious in datacentres due to the large amount of electricity consumption, ultimately emission of $\mathrm{CO}_{2}$ lead to the consequences of high electricity consumption. These processes had direct affect on the environment and human body, according to report [1] approximately 4.5 million people are dead all over world in 2016 by cause of air pollution. Now we needed such technologies that protect our environment. These technologies are known as a green cloud computing or green computing. We use green computing technology to reduce the energy consumption and CO2 emission [2]. Green cloud computing is work efficiently and effectively [3]. In recent year internet access have increased, as a result carbon emission also increased, here are some examples: watching videos on YouTube for 10 minutes can emit $1 \mathrm{~g}$ of $\mathrm{CO}_{2}$, Google search emits $0.2 \mathrm{~g}$ of $\mathrm{CO}_{2}$, and using Gmail account for a year emits $1200 \mathrm{~g}$ of $\mathrm{CO}_{2}$. Many companies have already moved on towards green cloud computing, namely: Google, Facebook, SAP, IBM, and Apple etc. Be clear that Google is declared $100 \%$ green company in 2017 using renewable resources [4].

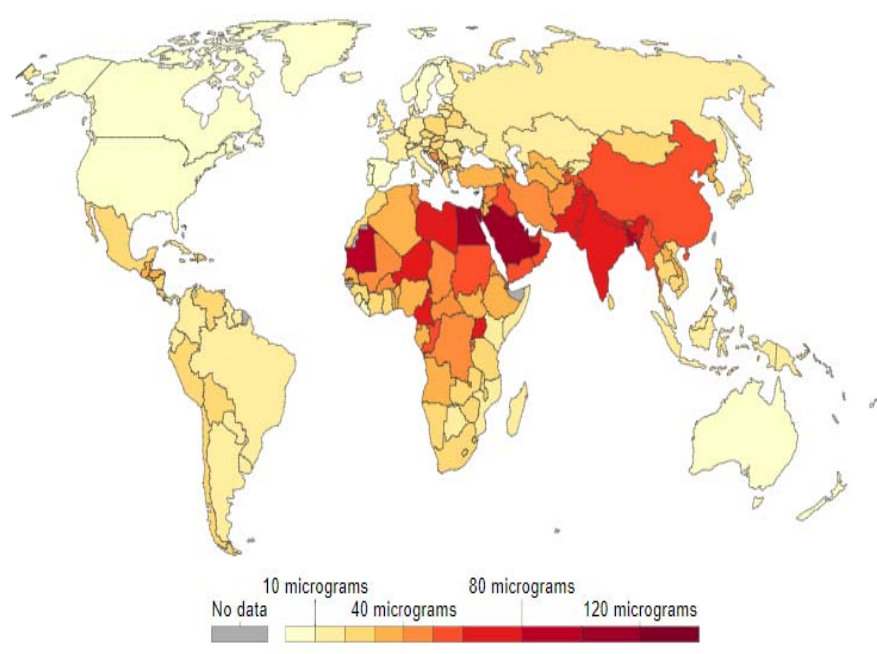

Figure 1: World air pollution weight [5].

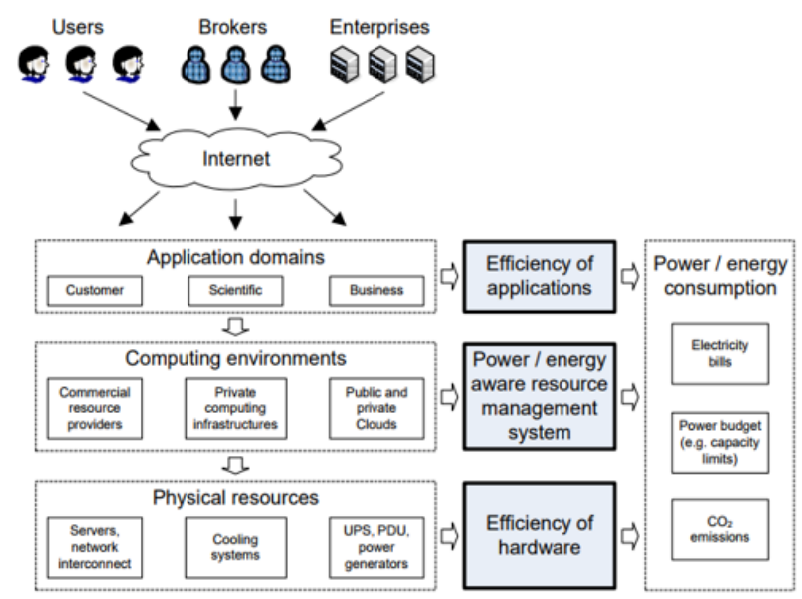

Figure 2: Energy consumption at different level [6]. 


\section{IMPACT OF GREEN HOUSE GASES ON NATURE}

- Electricity usage: Here we take three parameters of online activities: (1) Video Streaming, (2) Internet Surfing, and (3) Generation of Spam. These three activities produce 38 million tons of $\mathrm{CO}_{2}$ every year, according to an article [7] published in 2014 By Max Smolaks, he predicts the number of datacentres in the world will reach at 8.6 million in 2017, if this prediction is correct still one can't imagine the amount of electricity consumption and $\mathrm{CO}_{2}$ emission by 8.6 million datacenter, keeping in mind that all datacentres are running around the clock whole year. A datacentre consumes $6 \%-12 \%$ energy for computing purposes and rest of the energy goes towards maintaining datacentre temperature to keep it cool[8].

- Manufacturing process: the constructions of computing devices are using various types of toxic materials like mercury, lead, and cadmium. It is not problematic unless external harm on device is caused, which can produce harmful gases. These gases are very dangerous for ecosystem[9].

- Fossil Fuel: everyday pollution is increasing and destroying our ecosystem massively. Almost every country is burning fossil fuel such as coal, natural gases, and petroleum. These things produces $\mathrm{CO}_{2}$ in atmosphere. It is directly affected the environment and human beings. [10].

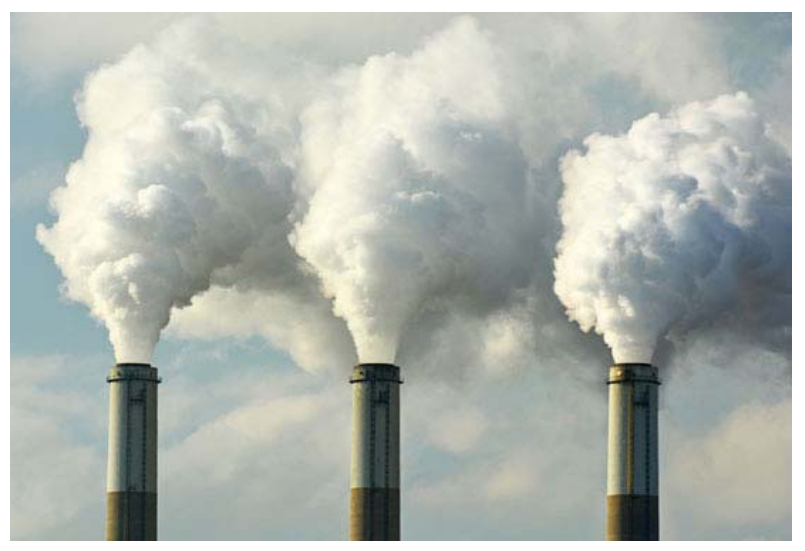

Figure 3: Burning fossil fuels [11].

\section{IMPORTANCE AND NEED OF GREEN COMPUTING}

As the time passes by, our environment is getting more polluted causing direct effect on our health. One of the major reasons of polluted environment is datacentre, already we have discussed above how datacentres are harmful for the environment and for the human health. Here we will talk about Electronic Waste, because E-Waste also responsible for pollution, so the question is what is E-Waste? All those machineries that are not in use anymore are called E-Waste, for example, our old computers, mobiles, laptops, and other electronic gear. When we crush and throw out our gadgets outside of our houses, it causes leak of some toxic gases and metal into the groundwater and the atmosphere, especially when E-Wastes are warm, they discharge toxic chemicals, these chemicals spare with the air, become the reason of the impairment in the atmosphere. Electronic Waste is growing exceedingly every year. You can get the idea how much EWaste has destroyed in the US only, according to a report, in the United States about 24 million computers have become antiquated yearly, only 3.3 million computers goes for recycling, approximately more than 20 million computers declare as waste material [12] [13]. Here the biggest question is what will be the consequences of these E-Waste on human health and this environment? That's why green computing and green technologies are very important to our society, to our nation, to our health and last but not the least to our environment.

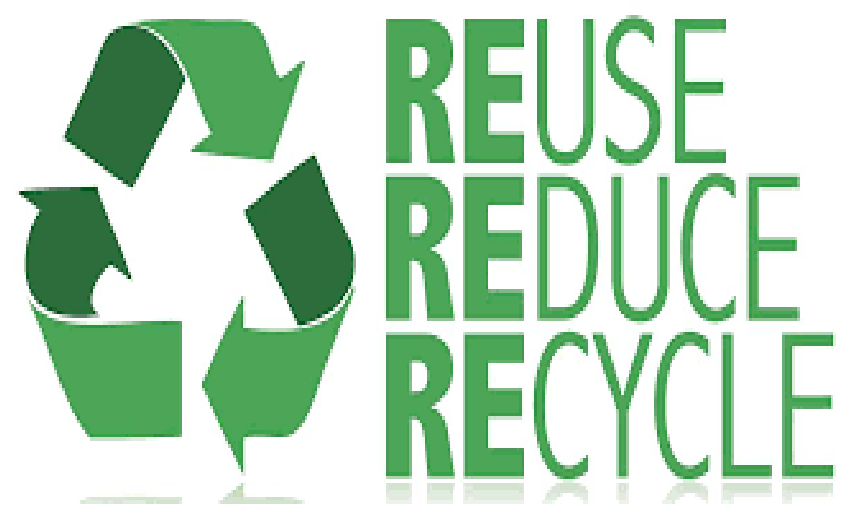

Figure 4: Recycling [14].

\section{MEASUREMENT OF ELECTRICITY IN A DATA CENTER}

The measurement techniques are listed below which help compute the electricity consumption in datacenter, such as: a)Energy Reuse Factor (ERF), b) Power usage Effectiveness (PUE), c) DataCenter infrastructure Efficiency (DCiE), d) Water usage Effectiveness (WUE), e) Carbon usage Effectiveness (CUE), f) DataCenter Productivity (DCP), g) Green Energy coefficient (GEC), h) Thermal Design Power (TDP), i)Compute Power Efficiency (CPE),j) Performance per Watt (PpW) [2].

\section{A. Energy Reuse Factor (ERF)}

Energy Reuse Efficiency (ERE) is a reusable form of the energy from a datacenter to Power Usage Effectiveness (PUE). The amount of energy reused from outside of the data center to effect ERE. The ERF must be used to calculate ERE from the site PUE. The ERE and ERF are defined as:[2][15].

$$
\begin{gathered}
\text { ERE }=\text { Total Energy }- \text { Reusable Energy }(1) \\
E R F=\frac{\text { Energy Reused }}{\text { Total Facility Power }}(2) \\
\text { ERE }=(1-\text { ERF }) * \text { PUE }(3)
\end{gathered}
$$

\section{B. Power usage Effectiveness (PUE)}

Effectiveness (PUE) is a calculation metric, used for measuring the energy efficiency of data center and physical infrastructure such as power and cooling equipment. The PUE described as the ratio of overall 
consumed power from the data center to the total consumed electricity by IT devices. Elaborately as:

$$
P U E=\frac{\text { Total Data center Power }}{\text { IT Devices Power }}
$$

When the efficiency of data center PUE lies between 1.3 to 3.0 approximately for all data center, if the measuring of PUE is 1.0 it is measured as excellent.[2][16].

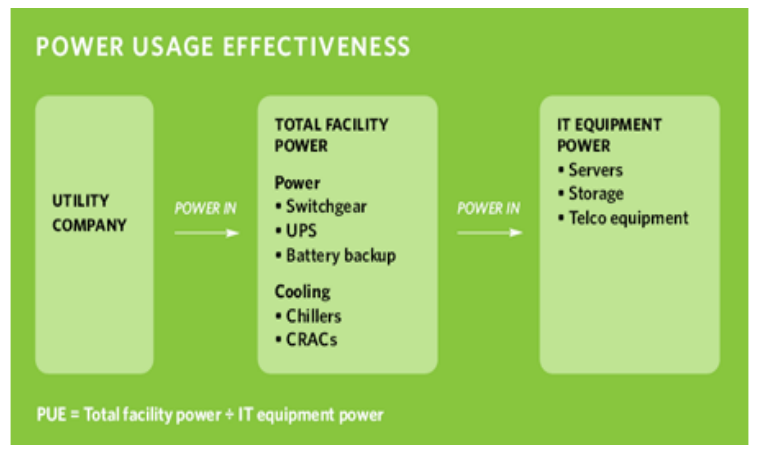

Figure 5: Power Usage Effectiveness [16].

\section{Data Center infrastructure Efficiency (DCiE)}

DCiE calculates the energy efficiency and improve the performance of a data center.DCiE and PUE are most popular techniques to measure the electricity of the data. It is defined as:[2][17]

$$
\begin{gathered}
\text { DCiE }=\frac{1}{\mathrm{PUE}} \\
=\frac{\text { IT Devices Power }}{\text { Total Data center Power }}
\end{gathered}
$$

Table 1:- Calculate PUE and DCiE [18].

\begin{tabular}{|c|c|c|}
\hline PUE & DCiE & Level of Efficieny \\
\hline 3.0 & $33 \%$ & Very Inefficient \\
\hline 2.5 & $40 \%$ & Inefficient \\
\hline 2.0 & $50 \%$ & Average \\
\hline 1.5 & $67 \%$ & Efficient \\
\hline 1.2 & $83 \%$ & Very Efficient \\
\hline
\end{tabular}

\section{Water Usage Effectiveness (WUE)}

WAE is helping to calculate yearly water requirement for cooling a data center and other equipment in a building. It is defined as:[2][19].

$$
W U E=\frac{\text { Water Used Yealy }}{\text { EIT }}
$$

\section{E. Carbon Usage Effectiveness (CUE)}

CUE is measured $\mathrm{CO} 2$ emission. CUE defined as:

$$
C U E=\frac{\mathrm{Eco} 2}{\mathrm{EIT}}
$$

Where

Eco2 $=$ Total CO2 emission from total energy consumed by the data center.

EIT $=$ Total energy consumed by IT Devices.[2]

\section{F. Data Center Productivity (DCP)}

DCP is helping to calculate the valuable amount of a work done by the data center. It is defined as:

$$
D C P=\frac{\text { Valuable Work Done }}{\text { Tresource }}
$$

Where

Tresource $=$ Total resource which was taken to done this valuable work. [2]

\section{G. Green Energy coefficient (GEC)}

GEC is a main focus to use renewable energy like wind energy, hydroelectric energy or solar energy. It makes data centers Eco friendly. GEC defined as:[2]

$$
\text { GEC }=\frac{\text { Green Power }}{\text { Total Facility Power }}
$$

\section{H. Thermal Design Power (TDP)}

The Thermal Design Point, is the maximum power consumption by a CPU or GPU in a real-time applications, the maximum power wasted by the device is called as TDP.[2]

\section{Compute Power Efficiency (CPE)}

The Computing Efficiency (CPE), is used to measure the efficiency during the idle state of devices. The CPE can be formulated as: [2]

$$
\begin{gathered}
C P E=\frac{\text { IT Devices Utilization }}{\text { PUE }} \\
C P E=\frac{\text { IT Devices Utilizatipon X IT Devices Power }}{\text { Total Data center Power }}
\end{gathered}
$$

\section{J. Performance per Watt (PpW)}

It computes the energy efficiency of an individual and computer hardware or computer architecture. According to Wikipedia” it measures the rate of computation that can be delivered by a computer for every watt of power consumed" [20]. Usually, it is measured in Floating-point operations per second (FLOPS) and Million instructions per second (MIPS).[2] 


\section{SOME METHOD OF ENERGY SAVING FROM CLOUD COMPUTING}

1)Hardware energy saving

a) The datacentre is a place where different type of computers are kept and placed, every computer needs a multiprocessor and its own memory, these equipment consumed lots of energy, that's why this issue has been a focal point between researchers from the last few years, they have suggested few ideas to improve the efficiency of energy saving. Computer architecture is helping to describe the performances, grouping, and implementation of computer system effectively such as Power Consumption Ratio (PCR) using the general purpose processor in the main control unit and the dedicated processor are increasing the performance and its help to reduce the energy consumption of the structure. Here, David Andersen, Suggested the FAWN (Fast Array of Wimpy Nodes) concept, this concept helps in low power consumption cluster system structure for large-scale dataintensive applications. It is increases the performance of the system also consumed low energy [21].

b) Energy Saving by Controling the Temperature: continous usage of 24x7 datacentre's temperature reaches the maximum heat, to overcome this problem we have two ways: (1) The first one is Green Computing, this technique has been designed to reduce the energy consumption and heating issues of the datacentre's hardwares, green technology are also responsible to maintain the cooling system of the datacentre. (2) The other way is Intelligent Temprature Control (ITC), heating increases because of Temprature Shock, the roll of ITC is to reduce this problem intelligently using Artificial Intelligence and Machine Learning techniques. Researchers found some sufficient methods, for instance. They are recommending SSDs instead of HDDs for storage solution. Rack Server Systems instead of Blade Server System, because BSS generates more heat, and it is costlier than RSS [22]. IBMs "Electron Spin" storage technology, APC "Thermal Channel Sealing System", and many others. To achieve desired results, we should install the Thermal Sensor around the hardwares for real time observation and Intelligent Dynmic Cooling System that will observe the current hardware state and work accordingly[21].

\section{2) Software enegry saving}

a) Using compiling technology for energy saving: Compiler optimization is the most critical method of compiler design, mainly in high-performance compiler design. Nowadays people are using an advanced compiler optimization technique, this technique helps to improve the performance of the compiler and overcome the energy consumption, research is not focused only on optimization of the application capacity, it can also measure the behavior of application program to overcome system or processor operation energy consumption. Zhao Rongcai put forward a model and method, it gives a handto overcome the execution frequency to cut down the power consumption in multithreading system design [21].

b) Energy saving method for application software power: The improvement work of software system power executed in the source program structure level, already cycle structure optimizationtechnique are related to this field, that has been executed in the compiler and achieved the best power optimization effect using some already existing algorithms, like improving data structure, compression data storage space, decrease the repeated calculation and algorithm redundancy, the greedy method,etc.,if measure the power optimization algorithm level, it can overcome the space complexity and time complexity of the algorithm for software power consumption[21].

\section{c) Energy saving method of system software involves} three facts:

2.c.1 Dynamic energy consumption management of operating system: Dynamic energy consumption management implies that the operating system regulates system unit power consumption dynamically according to system operation state, which might offer full play to the energy-saving features of the low-power hardware. Analysis of this space includes memory allocation strategy, supported energy consumption perception, dynamic adjustment mechanism, and supported load perception model of memory chip energy consumption.

2.c.2 Energy-efficient scheduling between nuclear: Energy-efficient scheduling between nuclear suggests energy saving scheduling strategy in the polynuclear system. Wang Jing analyzed thread scheduling strategy and resource classification mechanism for reducing resource rivalry and mentioned the possible future research direction of development.

2.c.3 Equipment resources management: this is a management technique for dynamic resource division and the combination of variable size, will optimum the environment for every individual computing task.[21]

\section{3) Energy Saving method of Virtual Machine Manager:}

Energy saving methods of a virtual machine manager involve the following conditions, energy consumption management interface support, energy consumption management framework, desktop-class virtual machine energy saving, and much more. Stoess, proposed a framework for energy management in virtualized servers, and its help to provide resources statistics and allocation mechanism of the division of energy consumption and energy consumption perception. In a collaborative model of energy management and task scheduling strategy among the operating systems, virtual machine manager and upper-level application of hardware can be studied for green energy saving strategy of the cloud computing platform. Ye Kejiang, introduces four conditions: the energy consumption measurement, energy consumption modeling, energy consumption management and realization mechanism, energy consumption management optimization algorithm, to virtualize cloud computing platform for energy consumption management. Here also using put the forward energy consumption model of two key technologies [21].

\section{4) Energy saving method of Network environment:}

Cloud computing is a branch of networking and completely rely on the Internet, if we implement energy preserving approaches on the network surrounding it can show us the enormous outcome in a positive manner. Currently green agent with the help of dormancy mechanism is a comman energy utilization and consumption technique. This combination 
works by modifying and adjusting the network topological structure. Many algorithm and protocol of the network does not fulfill the energy saving requirement, on the contrary these algorithms consume more energy, for example: Retransmission mechanism in Transmission Control Protocol (TCP), CSMA/CD and CSMA/CA in a wireless network, etc. This technique can also be used in thermal sensor network of processor, it helps to avoid unnecessary energy consumption.[21].

\section{VI.CONCLUSION AND FUTURE SCOPE}

Our main motto is to abridge the pollution from the earth, there are many reasons of the polluted environment as we have discussed above, being a computer science student it is our responsibility to defend our field, and we want our least hand to be environmentally polluted. We have explained different types of techniques that can be implemented in data centres, also we have reviewed about the importance of green computing and the impact of greenhouse gases on nature. This paper is a very small effort towards green and neat atmosphere. In future we shall use artificial intelligence and machine learning algorithms in datacenters to obtain more excellent results, as Google recently started using AI techniques.

\section{ACKNOWLEDGMENT}

I would like to pay special thankfulness,Mr.Abdul Majid Farooqi for devoting his time in discussing ideas with me and giving his invaluable feedback. His encouragement made it possible to achieve the goal.

\section{REFERENCES:}

[1] https://ourworldindata.org/air-pollution\#absolute-number-ofdeaths-from-air-pollution , retrieved date: 07/11/2017.

[2] Abdul Majid Farooqi,Md. TabrezNafis, KafiyahUsvub "Comparative Analysis of Green Cloud Computing," International Journal of Advanced Research in Computer Science, vol. 8, no. 2, pp. 56-60, 2017.

[3] S. R. Hussein, Y. Alkabani, and H. K. Mohamed, "Green cloud computing: Datacenters power management policies and algorithms," Proc. 2014 9th IEEE Int. Conf. Comput. Eng. Syst. ICCES 2014, pp. 421-426, 2014.

[4] https://cloud.google.com/environment/, Cloud.googl.com, GOOGLE CLOUD AND THE ENVIRONMENT, Retrieved Date: $20 / 12 / 2017$.

[5] https://ourworldindata.org/air-pollution\#empirical-view ,Retrieved Date: 20/12/2017

[6] https://www.google.co.in/search?hl=en\&biw=1292\&bih=677 \&tbm=isch\&sa=1\&ei=e6doWuvFHIvyvgTSlaz4DQ\&q=+clo ud+energy+consumption+at+different+level\&oq=+cloud+ene rgy+consumption+at+different+level\&gs_l=psyab.3...17711.18369.0.19194.5.5.0.0.0.0.266.266.21.1.0....0...1c.1.64.psyab..4.0.0....0.3HqtFjazHyw\#imgrc=DaNBFU0d33ff3M:public ation date: 3/22/2011, Retrieved date: 07/11/2017.
[7] http://www.datacenterdynamics.com/content-tracks/colocloud/number-of-data-centers-to-decrease-after-

2017/91495.fullarticle, Number of data centers to decrease after 2017, By Max Smolaks, Publication Date: 11/11/2014.

[8] https://getvoip.com/blog/2015/01/19/environmental-impactof-cloud/The Incredible Environmental Impact of Cloud Technology 2015, say study, publication date: 1/19/2015, retrieved date: 07/11/2017.

[9] A. Chopra, S. Sharma, and V. Kadyan, "Need of Green computing to improve environmental condition in current era,” pp. 3209-3212, 2016.

[10] https://sciencing.com/greenhouse-gases-bad-earth23688.html, Sciencing.com, How Are Greenhouse Gases Bad for the Earth?, By Sophie Johnson, Publication Date: 04/24/2017,Retrieved date: 07/11/2017.

[11] https://www.google.co.in/search?biw=1175\&bih=615\&tbm=i sch\&sa=1\&ei=K9NBWrX_EMbevASFnKdQ\&q=fossil+fuels \&oq=fossil\&gs_l=psyab.3.0.0i67k1j0l2j0i67k1j0j0i67k1l3j0l 2.233650.239828.0.241182.36.14.1.0.0.0.389.1909.2-

3j3.7.0.......1c.1.64.psy\#imgrc=PgyR-O1ozLuPJM:Retrieved date: 20/12/2017.

[12] https://www.ukessays.com/essays/informationtechnology/the-importance-of-green-computing-informationtechnology-essay.php

The Importance of Green Computing Information Technology Essay, Published: 23rd March, 2015, retrieved date: 12/11/2017

[13] https://www.allgreenrecycling.com/effects-of-e-waste-on-ourenvironme/ EFFECTS OF E-WASTE ON OUR ENVIRONMENT, Retrieved Date: 20/12/2017.

[14] https://www.google.co.in/search?biw=1175\&bih=571\&tbm=i sch\&sa=1\&ei=HdRBWtfCEoH6vgSBoI3YCw\&q=recycling \&oq=recycli\&gs_l=psy-

ab.3.0.0110.407083.411624.0.413975.19.12.0.1.1.0.368.940.21j2.4.0....0...1c.1.64.psy-

ab..14.4.969.0..0i67k1.524.OksdU_9u6Cc.Retrieved Date: $10 / 12 / 2017$

[15] M. Wahlroos, M. Pärssinen, S. Rinne, S. Syri, and J. Manner, "Future views on waste heat utilization - Case of data centers in Northern Europe,” Renew. Sustain. Energy Rev., vol. 82, no. July, pp. 1749-1764, 2018

[16] http://searchdatacenter.techtarget.com/definition/powerusage-effectiveness-PUE,TechTargetretrieveddate: 07/02/2018

[17] https://en.wikipedia.org/wiki/Data_center_infrastructure_effic iencyWikipediapublication date: 29/01/2018, retrieved date: 07/02/2018.

[18] http://www.42u.com/measurement/pue-dcie.htm, Solution for the next Generation data Center ,retrieved date: 07/02/2018.

[19] http://searchdatacenter.techtarget.com/definition/WUE-waterusage-effectiveness,TechTargetretrieved date: 07/02/201

[20] https://www.greenbiz.com/blog/2011/07/27/4-reasons-whycloud-computing-also-green-solution, Greenbiz.com, 4 Reasons Why Cloud Computing is Also a Green Solution, Published date 27/07/2017, retrieved date: 07/11/2017.

[21] L. Gong, J. Xie, X. Li, and B. Deng, "Study on Energy Saving Strategy and Evaluation Method of Green Cloud Computing System,” pp. 483-488, 2013.

[22] https://www.tuangru.com/the-pros-and-cons-of-blade-serversvs-rack-servers/ The Pros And Cons Of Blade Servers Vs. Rack Servers, by Rajan, Publication Date: 13/08/2014. 